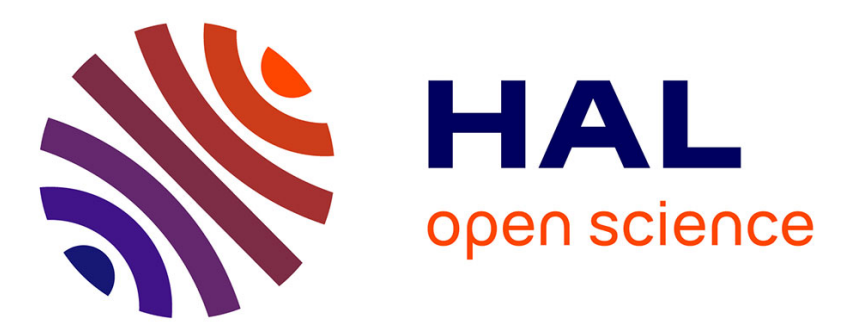

\title{
A Rigidity-Based Decentralized Bearing Formation Controller for Groups of Quadrotor UAVs
}

Fabrizio Schiano, Antonio Franchi, Daniel Zelazo, Paolo Robuffo Giordano

\section{To cite this version:}

Fabrizio Schiano, Antonio Franchi, Daniel Zelazo, Paolo Robuffo Giordano. A Rigidity-Based Decentralized Bearing Formation Controller for Groups of Quadrotor UAVs. IEEE/RSJ International Conference on Intelligent Robots and Systems, IROS 2016, Oct 2016, Daejeon, South Korea. pp.50995106. hal-01348543

\section{HAL Id: hal-01348543 https://hal.science/hal-01348543}

Submitted on 25 Jul 2016

HAL is a multi-disciplinary open access archive for the deposit and dissemination of scientific research documents, whether they are published or not. The documents may come from teaching and research institutions in France or abroad, or from public or private research centers.
L'archive ouverte pluridisciplinaire HAL, est destinée au dépôt et à la diffusion de documents scientifiques de niveau recherche, publiés ou non, émanant des établissements d'enseignement et de recherche français ou étrangers, des laboratoires publics ou privés. 


\title{
A Rigidity-Based Decentralized Bearing Formation Controller for Groups of Quadrotor UAVs
}

\author{
Fabrizio Schiano, Antonio Franchi, Daniel Zelazo, and Paolo Robuffo Giordano
}

\begin{abstract}
This paper considers the problem of controlling a formation of quadrotor UAVs equipped with onboard cameras able to measure relative bearings in their local body frames w.r.t. neighboring UAVs. The control goal is twofold: $(i)$ steering the agent group towards a formation defined in terms of desired bearings, and $(i i)$ actuating the group motions in the 'nullspace' of the current bearing formation. The proposed control strategy relies on an extension of the rigidity theory to the case of directed bearing frameworks in $\mathbb{R}^{3} \times \mathcal{S}^{1}$. This extension allows to devise a decentralized bearing controller which, unlike most of the present literature, does not need presence of a common reference frame or of reciprocal bearing measurements for the agents. Simulation and experimental results are then presented for illustrating and validating the approach.
\end{abstract}

\section{INTRODUCTION}

The problem of formation control of multiple mobile robots has been extensively studied over the last decade in the robotics and control communities. The basic goal of most formation controllers is to coordinate a robot team in order to achieve some desired spatial arrangement. Most formation control schemes differ in their assumptions about, e.g., the robot mobility (planar robots, aerial vehicles, possible presence of non-holonomic constraints or underactuation), the employed sensing technology (local measurements from onboard sensors, or absolute/global measurement from centralized facilities) and overall architecture (centralized vs. decentralized coordination schemes).

A challenging scenario that still motivates considerable research efforts is that of decentralized formation control of mobile robots based on only local sensing, in which the robots are assumed to only be able to obtain relative measurements with respect to other robots in the group. Common examples of relative sensing include range sensors for retrieving inter-robot distances, or bearing sensors (such as cameras) for obtaining the bearing angle to other robots within visibility. The use of local sensing also entails the possible lack of a common reference frame to which all the individually collected measurements and control actions can be expressed, a limitation that poses additional challenges for solving the formation control problem [1]-[3]. These scenarios are motivated by the goal of deploying highly autonomous multi-robot teams in 'non-trivial' environments

F. Schiano is with Inria at Irisa Rennes, Campus de Beaulieu, 35042 Rennes Cedex, France fabrizio.schianodinria.fr

A. Franchi is with LAAS-CNRS, Université de Toulouse, CNRS, Toulouse, France, afranchielaas.fr

D. Zelazo is with the Faculty of Aerospace Engineering at the TechnionIsrael Institute of Technology, Haifa, Israel dzelazo@technion.ac.il.

P. Robuffo Giordano is with CNRS at Irisa and Inria Rennes, Campus de Beaulieu, 35042 Rennes Cedex, France prg@irisa.fr. (e.g., inside buildings, underwater, underground, or even in deep space) where centralized sensing facilities, such as GPS, are not available, and the robots can only rely on their 'local skills' (local sensing and computing power, and local communication with neighboring agents).

In all these cases, the correct theoretical framework for analyzing and controlling the geometrical properties of robot formations defined in terms of relative measurements has proven to be the theory of formation rigidity [4]. Rigidity theory has found a large number of applications in the context of formation control based on, e.g., relative distance measurements [5]-[7] and relative bearing measurements [8]-[13]. Rigidity theory plays also a pivotal role in the dual problem of cooperative localization from local relative measurements [7], [14]-[17], since rigidity of the formation is a necessary requirement for recovering from the available relative measurements a consistent solution of the localization problem in a common shared frame.

When exploiting cameras as the onboard sensing modality, rigidity-based bearing formation control has received an increasing attention over the last years [8], [10]-[12], mainly differing in the assumptions and/or simplifications taken at the design stage. For instance, the authors of [8], [10] have considered the formation control of planar kinematic agents by assuming a common reference frame and an undirected topology for the measurement graph (i.e., all measurements are assumed to be reciprocal), and [12] has extended these results to arbitrary dimensions. The work [11] has instead dropped the assumption of a common reference while, however, retaining that of an undirected measurement topology.

Nevertheless, when addressing visual-based formation control in GPS-denied environments, the assumptions of a common reference frame and of an undirected topology for the measurement graph can be hard to realize. For instance, the requirement of keeping constant mutual visibility among all robot pairs can easily become unfeasible because of the limited camera fov, and likewise for the possible presence of a common frame shared by a group of robots that has only access to local relative measurements. It is then important to investigate possible bearing formation control strategies that can relax as much as possible these assumptions.

In this respect, to the best of our knowledge, the only decentralized bearing formation controller that does not require presence of a common frame and of an undirected sensing topology has been proposed in [18] by exploiting the (bodyframe) measured bearings and a single distance measurement among an arbitrary pair of robots. However, the machinery presented in [18] requires a very special structure for the cho- 
sen measurement graph which, among others, must contain two special agents able to measure and to be measured by any other agent in the group. While instrumental, this choice is also unnecessarily overconstraining since any bearing rigid topology (with, in general, far less constraining requirements than the ones exploited in [18]) would still allow solving a bearing formation control problem.

The goal of this work is to then generalize the ideas of [18] by proposing a fully decentralized bearing formation controller that only requires presence of a generic (directed) bearing rigid topology. Furthermore, as in [18], the proposed control strategy is also complemented with the possibility of steering the quadrotor group along all the bearing-preserving motion directions: these can be shown to consist of a collective translation, an expansion with respect to a reference point, and a coordinated rotation relative to a reference vertical axis. This possibility is particularly useful when, for instance, needing to collectively steer the quadrotor group for navigation or exploration purposes while maintaining a desired bearing formation optimized for the task at hand.

These two goals (bearing formation stabilization and group collective steering) are here achieved under a minimal number of assumptions compared to the existing literature, in particular: $(i)$ the quadrotors are only assumed able to collect bearing measurements and to impose motion commands in their (local) body-frames (which do not need to be aligned or 'coordinated' in some special ways), ( $i i)$ the bearing measurements are not necessarily required to be reciprocal, and the resulting (directed) sensing topology has no special constraints (apart from the 'necessary condition' of yielding a rigid bearing formation), (iii) a single (but arbitrary) quadrotor pair is additionally assumed able to measure its interdistance (needed to retrieve the correct scale of formation which would be, otherwise, unobservable from only bearing measurements). Finally, we also provide an experimental validation of the overall approach by means of a group of four quadrotor UAVs.

The rest of the paper is organized as follows: Sect. II reviews the main modeling assumptions and recaps some key notions of bearing rigidity and related concepts. Then, Sect. III illustrates the proposed bearing formation control strategy for robots evolving in $\mathbb{R}^{3} \times \mathcal{S}^{1}$, which is representative of the quadrotor case. Subsequently, Sect. IV reports the simulation results obtained by considering a group of quadrotor UAVs while Sect. V presents the experiments with real robots. Finally, Sect. VI concludes the paper and discusses some future directions.

\section{PRELIMINARIES}

\section{A. Agent model}

In this work we consider a group of $N$ quadrotor UAVs equipped with onboard Inertial Measurement Units (IMUs) and (calibrated) cameras, and able to exchange data over a radio communication channel. Let $\mathcal{W}$ : $\left\{\boldsymbol{O}_{\mathcal{W}}, \boldsymbol{X}_{\mathcal{W}}, \boldsymbol{Y}_{\mathcal{W}}, \boldsymbol{Z}_{\mathcal{W}}\right\}$ represent the world frame. Following [18], [19], we consider the following simplified kinematic model for the $i$-th quadrotor

$$
\left(\begin{array}{c}
\dot{\boldsymbol{p}}_{i} \\
\dot{\psi}_{i}
\end{array}\right)=\left(\begin{array}{cc}
\boldsymbol{R}_{i} & \mathbf{0} \\
\mathbf{0} & 1
\end{array}\right)\left(\begin{array}{c}
\boldsymbol{u}_{i} \\
w_{i}
\end{array}\right)
$$

where $\boldsymbol{p}_{i} \in \mathbb{R}^{3}$ is the robot $3 \mathrm{D}$ position in the world frame, $\psi_{i} \in \mathcal{S}^{1}$ is the yaw angle and $\boldsymbol{R}_{i}=\boldsymbol{R}_{z}\left(\psi_{i}\right) \in$ $S O(3)$ is the canonical rotation around the world $\boldsymbol{z}$-axis. The quantities $\boldsymbol{u}_{i} \in \mathbb{R}^{3}$ and $w_{i} \in \mathbb{R}$ are the body-frame linear velocity and yaw rate which are assumed to be known and controllable. We stress that the (absolute) yaw angle $\psi_{i}$ is not considered as an available quantity to the $i$-th quadrotor ${ }^{1}$. As a consequence, the $N$ quadrotors are not assumed to share, as a group, a common (global) reference frame where to express local measurements and control inputs.

The agent relative bearing (from agent $i$ to agent $j$ and expressed in the body frame of agent $i$ ) is then defined as the $3 \mathrm{D}$ unit vector

$$
\boldsymbol{\beta}_{i j}=\boldsymbol{R}_{i}^{T} \frac{\boldsymbol{p}_{j}-\boldsymbol{p}_{i}}{\left\|\boldsymbol{p}_{j}-\boldsymbol{p}_{i}\right\|} \in \mathbb{S}^{2} .
$$

This agent relative bearing $\boldsymbol{\beta}_{i j}$ can be retrieved by 'derotating' the actual bearing measurement among quadrotors $i$ and $j$ (e.g., from an onboard camera) by the roll/pitch angles which can be typically measured by exploiting the onboard IMU, see also [18].

The control strategy discussed in the next Sections will then be based on model (1) and will assume that each agent $i$ can measure the (body-frame) bearing vector $\boldsymbol{\beta}_{i j}$ with respect to other neighboring agents.

\section{B. Directed Bearing Rigidity in $\mathbb{R}^{3} \times \mathcal{S}^{1}$}

We now recap some relevant definitions and properties of directed bearing formations and bearing rigidity in $\mathbb{R}^{3} \times \mathcal{S}^{1}$.

Let $\mathcal{G}=(\mathcal{V}, \mathcal{E})$ be a directed graph, where $\mathcal{V}=\{1 \ldots N\}$ is the vertex set and $\mathcal{E} \subseteq \mathcal{V} \times \mathcal{V}$ the edge set [20]. Presence of an edge $e_{k}=(i, j)$ in $\mathcal{E}$ represents the possibility for agent $i$ to measure the relative bearing $\boldsymbol{\beta}_{i j}$ (2) to agent $j$. Graph $\mathcal{G}$ is designed as directed as we do not require, in general, reciprocity of the relative bearing measurements.

Let now $\boldsymbol{q}=(\boldsymbol{p}, \boldsymbol{\psi}) \in\left(\mathbb{R}^{3} \times \mathcal{S}^{1}\right)^{N}$ represent the configuration of $N$ agents (1), with $\boldsymbol{q}_{i}=\left(\boldsymbol{p}_{i}, \psi_{i}\right) \in \mathbb{R}^{3} \times \mathcal{S}^{1}$ being the configuration of the $i$-th agent in the group. A framework (or also formation) [19], [21], [22] is the pair $(\mathcal{G}, \boldsymbol{q})$ where $\boldsymbol{p}_{i}: \mathcal{V} \rightarrow \mathbb{R}^{3}$ and $\psi_{i}: \mathcal{V} \rightarrow \mathcal{S}^{1}$ map each vertex in $\mathcal{V}$ to a point $\left(\boldsymbol{p}_{i}, \psi_{i}\right)$ in $\mathbb{R}^{3} \times \mathcal{S}^{1}$. Two frameworks $(\mathcal{G}, \boldsymbol{q})$ and $\left(\mathcal{G}, \boldsymbol{q}^{\prime}\right)$ are denoted bearing equivalent if $\boldsymbol{\beta}_{i j}(\boldsymbol{q})=\boldsymbol{\beta}_{i j}\left(\boldsymbol{q}^{\prime}\right) \forall(i, j) \in \mathcal{E}$ and bearing congruent if $\boldsymbol{\beta}_{i j}(\boldsymbol{q})=\boldsymbol{\beta}_{i j}\left(\boldsymbol{q}^{\prime}\right) \forall i, j \in \mathcal{V}, i \neq j$. A framework $(\mathcal{G}, \boldsymbol{q})$ is defined bearing rigid (or simply rigid in the following) if there exists a neighborhood $\mathcal{U}$ of $\boldsymbol{q}$ such that any framework $\left(\mathcal{G}, \boldsymbol{q}^{\prime}\right), \boldsymbol{q}^{\prime} \in \mathcal{U}$, that is bearing equivalent to $(\mathcal{G}, \boldsymbol{q})$ is also bearing congruent to $(\mathcal{G}, \boldsymbol{q})$. By extension, a framework is instead defined globally bearing rigid if $\mathcal{U}=\left(\mathbb{R}^{3} \times \mathcal{S}^{1}\right)^{N}$, i.e., if all frameworks which are bearing equivalent to $(\mathcal{G}, \boldsymbol{q})$

\footnotetext{
${ }^{1}$ Indeed, additional sensors, such as compasses, would be needed for obtaining a consistent yaw measurement for all the UAVs in group with, however, a typically limited reliability (e.g., compasses would fail to operate soundly indoor or close to strong magnetic fields).
} 
are also bearing congruent to $(\mathcal{G}, \boldsymbol{q})$. A non-rigid framework is also termed roto-flexible, and a framework $(\mathcal{G}, \boldsymbol{q})$ is said to be minimally rigid if $(\mathcal{G}, \boldsymbol{q})$ is rigid and the removal of any edge yields a roto-flexible framework.

The notion of bearing rigidity can also be characterized from an infinitesimal perspective by introducing the so-called directed bearing function and corresponding directed bearing rigidity matrix. The directed bearing function (bearing function from now on) associated to a framework $(\mathcal{G}, \boldsymbol{q})$ is the $\operatorname{map} \boldsymbol{\beta}_{\mathcal{G}}(\boldsymbol{q}):\left(\mathbb{R}^{3} \times \mathcal{S}^{1}\right)^{N} \rightarrow\left(\mathbb{S}^{2}\right)^{|\mathcal{E}|}$

$$
\boldsymbol{\beta}_{\mathcal{G}}(\boldsymbol{q})=\left[\boldsymbol{\beta}_{e_{1}}^{T} \ldots \boldsymbol{\beta}_{e_{|\mathcal{E}|}^{T}}^{T}\right]^{T}
$$

where the notation $e_{i} \in \mathcal{E}$ is used to represent a directed edge in the graph $\mathcal{G}$ according to any chosen labeling. The worldframe (directed) bearing rigidity matrix is the Jacobian of the bearing function with respect to the agent configuration $\boldsymbol{q}$, that is, the matrix

$$
\mathcal{B}_{\mathcal{G}}^{\mathcal{W}}(\boldsymbol{q})=\frac{\partial \boldsymbol{\beta}_{\mathcal{G}}(\boldsymbol{q})}{\partial \boldsymbol{q}} \in \mathbb{R}^{3|\mathcal{E}| \times 4 N} .
$$

Let $\mathcal{N}(\cdot)$ represent the null-space of a matrix. A framework $(\mathcal{G}, \boldsymbol{q})$ is said to be infinitesimally bearing rigid at some point $\boldsymbol{q}$ if $\mathcal{N}\left(\boldsymbol{B}_{\mathcal{G}}^{\mathcal{W}}(\boldsymbol{q})\right)=\mathcal{N}\left(\mathcal{B}_{\mathcal{K}_{N}}^{\mathcal{W}}(\boldsymbol{q})\right)$, with $\mathcal{K}_{N}$ being the complete directed graph. Otherwise a framework is said to be infinitesimally roto-flexible. Since it can be shown that $\operatorname{dim} \mathcal{N}\left(\mathcal{B}_{\mathcal{K}_{N}}^{\mathcal{W}}(\boldsymbol{q})\right)=5$, see, e.g., [18], it follows that a framework $(\mathcal{G}, \boldsymbol{q})$ in $\mathbb{R}^{3} \times \mathcal{S}^{1}$ is infinitesimally rigid if and only if $\operatorname{rank}\left(\mathcal{B}_{\mathcal{G}}^{\mathcal{W}}(\boldsymbol{q})\right)=4 N-5$. For infinitesimally rigid frameworks in $\mathbb{R}^{3} \times \mathcal{S}^{1}$, the 5 -dimensional null-space of the bearing rigidity matrix is also well-understood: it corresponds to the three rigid-body translations, a dilation relative to a reference point, and a coordinated rotation about a vertical axis passing through a reference point [18], [19], [22].

\section{DECENTRALIZED BEARING FORMATION CONTROL}

Consider a bearing rigid framework $(\mathcal{G}, \boldsymbol{q})$ in $\mathbb{R}^{3} \times \mathcal{S}^{1}$ consisting of $N$ agents with dynamics (1). Let $\boldsymbol{q}_{d}$ be a desired configuration such that $\left(\mathcal{G}, \boldsymbol{q}_{d}\right)$ is bearing rigid, and let $\boldsymbol{b}_{\mathcal{G}}^{d}=\boldsymbol{\beta}_{\mathcal{G}}\left(\boldsymbol{q}_{d}\right)=\left(\boldsymbol{\beta}_{e_{1}}^{d} \ldots \boldsymbol{\beta}_{e_{\mid} \mathcal{E} \mid}\right)$ be the corresponding desired value for the bearing function. Our goal is to design a decentralized bearing formation controller able to accomplish two distinct objectives.

A first objective is the bearing formation stabilization: by acting on the control inputs $\left(\boldsymbol{u}_{i}, w_{i}\right)$, the controller should steer the $N$ agents towards a configuration $\boldsymbol{q}^{*}$ equivalent to $\boldsymbol{q}_{d}$, i.e., such that $\boldsymbol{\beta}_{\mathcal{G}}\left(\boldsymbol{q}^{*}\right)=\boldsymbol{b}_{\mathcal{G}}^{d}$. Because of the framework rigidity, equivalence will also imply congruency with $\boldsymbol{q}_{d}$. Hence, the fulfillment of this first objective will ensure that $\boldsymbol{q}(t)$ reaches the (correct) desired shape modulo a possible translation, vertical rotation, and scaling (i.e., the motions spanning the null-space of the bearing rigidity matrix).

A second independent objective, 'orthogonal' to the first one, is the possibility to steer the agent group along the motion directions that do not affect the bearing rigidity function (for not interfering with the previous formation control goal). These are spanned by the null space of the rigidity matrix.

Finally, the formation controller should be decentralized and only based on information locally available or communicated by 1-hop neighbors.

\section{A. Rigidity-based control of bearing frameworks in $\mathbb{R}^{3} \times \mathcal{S}^{1}$}

Extending the results of [19] to the case of frameworks in $\mathbb{R}^{3} \times \mathcal{S}^{1}$, the $k$-th row block of the bearing rigidity matrix $\mathcal{B}_{\mathcal{G}}^{\mathcal{W}}$ in (3) associated to edge $e_{k}=(i, j)$ has expression

$$
\begin{gathered}
{\left[\begin{array}{c}
-\mathbf{0}-\underbrace{-\frac{\boldsymbol{P}_{i j} \boldsymbol{R}_{i}^{T}}{d_{i j}}}_{i}-\mathbf{0}-\underbrace{\frac{\boldsymbol{P}_{i j} \boldsymbol{R}_{i}^{T}}{d_{i j}}}_{j}-\mathbf{0}- \\
\cdots \underbrace{-\boldsymbol{S}_{i j}}_{3 N+i}-\mathbf{0}-
\end{array}\right] \in \mathbb{R}^{3 \times 4 N} .}
\end{gathered}
$$

Here, $d_{i j}=\left\|\boldsymbol{p}_{i}-\boldsymbol{p}_{j}\right\|, \boldsymbol{P}_{i j}=\boldsymbol{I}_{3}-\boldsymbol{\beta}_{i j} \boldsymbol{\beta}_{i j}^{T}$ is the orthogonal projector onto the orthogonal complement of $\boldsymbol{\beta}_{i j}$, and $\boldsymbol{S}=$ $\left.\left[\begin{array}{lll}0 & 0 & 1\end{array}\right]^{T}\right]_{\times}$where $[\cdot]_{\times}$indicates the usual skew-symmetric matrix operator.

We then note that the bearing rigidity matrix $\mathcal{B}_{\mathcal{G}}^{\mathcal{W}}$ is a function of interdistances, relative bearings, and absolute yaw rotations. Indeed, the bearing rigidity matrix relates changes in the bearing function $\boldsymbol{\beta}_{\mathcal{G}}$ to the world-frame velocities $\dot{\boldsymbol{q}}=(\dot{\boldsymbol{p}}, \dot{\boldsymbol{\psi}})$ of the framework

$$
\dot{\boldsymbol{\beta}}_{\mathcal{G}}=\mathcal{B}_{\mathcal{G}}^{\mathcal{W}}(\boldsymbol{q})\left[\begin{array}{c}
\dot{\boldsymbol{p}} \\
\dot{\boldsymbol{\psi}}
\end{array}\right]
$$

A first contribution of this work is the following, whose proof can be found in the Appendix.

Proposition 1. The null-space of the bearing rigidity matrix can be explicitly characterized as

$$
\begin{aligned}
\mathcal{N}\left(\boldsymbol{B}_{\mathcal{G}}^{\mathcal{W}}(\boldsymbol{q})\right) & =\operatorname{span}\left\{\left[\begin{array}{c}
\mathbf{1}_{N_{3}} \\
\mathbf{0}
\end{array}\right],\left[\begin{array}{l}
\boldsymbol{p} \\
\mathbf{0}
\end{array}\right],\left[\begin{array}{l}
\boldsymbol{p}^{\perp} \\
\mathbf{1}_{N}
\end{array}\right]\right\} \\
& =\operatorname{span}\left\{\boldsymbol{n}_{1}, \boldsymbol{n}_{2} \boldsymbol{n}_{3}\right\}
\end{aligned}
$$

where $\mathbf{1}_{N}$ is a vector of all ones of dimension $N, \mathbf{1}_{N_{3}}=$ $\mathbf{1}_{N} \otimes \boldsymbol{I}_{3}, \boldsymbol{p}^{\perp}=\left(\boldsymbol{I}_{N} \otimes \boldsymbol{S}\right) \boldsymbol{p}$, and $\otimes$ denotes the matrix Kronecker product.

Each null-space vector of this particular basis for $\mathcal{N}\left(\mathcal{B}_{\mathcal{G}}^{\mathcal{W}}(\boldsymbol{q})\right)$ represents one of the coordinated motions discussed above, namely, three translations along the world axes, an expansion about $\boldsymbol{O}_{\mathcal{W}}$, and a rotation about a vertical axis passing through $\boldsymbol{O}_{\mathcal{W}}$.

One can also define a body-frame bearing rigidity matrix: letting $\boldsymbol{u}=\left[\ldots \boldsymbol{u}_{i}^{T} \ldots\right]^{T} \in \mathbb{R}^{3 N}$ and $\boldsymbol{w}=\left[\ldots w_{i} \ldots\right]^{T} \in$ $\mathbb{R}^{N}$ represent the body-frame velocity inputs of the agent group, one has

$$
\dot{\boldsymbol{\beta}}_{\mathcal{G}}=\boldsymbol{B}_{\mathcal{G}}^{\mathcal{W}}(\boldsymbol{q})\left[\begin{array}{cc}
\operatorname{diag}\left(\boldsymbol{R}_{i}\right) & \mathbf{0} \\
\mathbf{0} & \boldsymbol{I}_{N}
\end{array}\right]\left[\begin{array}{c}
\boldsymbol{u} \\
\boldsymbol{w}
\end{array}\right]=\boldsymbol{B}_{\mathcal{G}}(\boldsymbol{q})\left[\begin{array}{c}
\boldsymbol{u} \\
\boldsymbol{w}
\end{array}\right] .
$$


The $k$-th row block of the body-frame bearing rigidity matrix $\mathcal{B}_{\mathcal{G}}(\boldsymbol{q})$ associated to edge $e_{k}=(i, j)$ is then

$$
\begin{gathered}
{\left[\begin{array}{c}
-\mathbf{0}-\underbrace{-\frac{\boldsymbol{P}_{i j}}{d_{i j}}}_{i}-\mathbf{0}-\underbrace{\frac{\boldsymbol{P}_{i j}{ }^{i} \boldsymbol{R}_{j}}{d_{i j}}}_{j}-\mathbf{0 -} \\
\cdots \underbrace{-\boldsymbol{S} \boldsymbol{\beta}_{i j}}_{3 N+i}-\mathbf{0}-
\end{array}\right] \in \mathbb{R}^{3 \times 4 N}}
\end{gathered}
$$

where ${ }^{i} \boldsymbol{R}_{j}=\boldsymbol{R}_{z}\left(\psi_{i j}\right)$ with $\psi_{i j}=\psi_{j}-\psi_{i}$.

The body-frame rigidity matrix is, again, a function of measured bearings $\boldsymbol{\beta}_{i j}$ and interdistances $d_{i j}$. However, contrary to the previous case, it is not a function of absolute yaw rotations $\psi_{i}$, but, instead, of relative orientations $\psi_{j}-\psi_{i}$ among neighboring agents. This fact will be important for the next developments.

Let now $\boldsymbol{e}_{F}(\boldsymbol{q})=\boldsymbol{b}_{\mathcal{G}}^{d}-\boldsymbol{\beta}_{\mathcal{G}}(\boldsymbol{q})$ denote the bearing formation control error to be regulated to zero for solving the first objective (formation stabilization). As shown in [22], minimization of $\left\|\boldsymbol{e}_{F}\right\|$ can be obtained by implementing the following scale-free controller based on the body-frame rigidity matrix,

$$
\left[\begin{array}{c}
\boldsymbol{u} \\
\boldsymbol{w}
\end{array}\right]=k_{c}\left[\begin{array}{cc}
\operatorname{diag}\left(d_{i j}\right) & \mathbf{0} \\
\mathbf{0} & \boldsymbol{I}_{N}
\end{array}\right] \boldsymbol{B}_{\mathcal{G}}(\boldsymbol{q})^{T} \boldsymbol{b}_{\mathcal{G}}^{d}, k_{c}>0
$$

that results in the $i$-th agent velocity command

$$
\left\{\begin{array}{rl}
\boldsymbol{u}_{i} & =-k_{c} \sum_{(i, j) \in \mathcal{E}} \boldsymbol{P}_{i j} \boldsymbol{\beta}_{i j}^{d}+k_{c} \sum_{(j, i) \in \mathcal{E}}{ }^{i} \boldsymbol{R}_{j} \boldsymbol{P}_{j i} \boldsymbol{\beta}_{j i}^{d} \\
w_{i} & =k_{c} \sum_{(i, j) \in \mathcal{E}} \boldsymbol{\beta}_{i j}^{T} \boldsymbol{S} \boldsymbol{\beta}_{i j}^{d}
\end{array} .\right.
$$

The reader is referred to [19] for an almost global stability proof for frameworks in $S E(2)$ that can be directly extended to the case under consideration. Furthermore, the centroid $\overline{\boldsymbol{p}}=\mathbf{1}_{N_{3}}^{T} \boldsymbol{p} / N$ and 'scale' $\boldsymbol{p}^{T} \boldsymbol{p}$ of the formation can be shown to be invariant under the action of (10).

It is worth noting that controller (10) has a decentralized structure depending only on the interaction graph $\mathcal{G}$ and on relative quantities. In particular, it does not require knowledge of any distance measurement (from which the term scale-free), nor knowledge of any common reference frame shared by the agent group. However, controller (10) requires communication among agents since, if there exists an edge $(j, i) \in \mathcal{E}$ (i.e., an agent $j$ is measuring agent $i$ ), agent $i$ needs to receive the bearing measurement $\boldsymbol{\beta}_{j i}$ and desired bearing $\boldsymbol{\beta}_{j i}^{d}$ from agent $j$ (second term of $\boldsymbol{u}_{i}$ ).

Furthermore, controller (10) also needs access to the relative orientation ${ }^{i} \boldsymbol{R}_{j}$ among neighboring pairs which is a quantity not available from direct measurements. However, if a framework is bearing rigid, all the relative orientations among agent pairs are univocally fixed by the existing interagent bearings constraints. Therefore, it is in principle conceivable to recover/estimate the relative rotations ${ }^{i} \boldsymbol{R}_{j}$ by processing the measured inter-agent bearings. This insight has, indeed, been exploited in [11], [18]. In [11] the assumption of reciprocal measurements for all agent pairs (undirected sensing graph $\mathcal{G}$ ) allows for an algebraic computation of all the needed relative orientations, and an analogous solution is exploited in [18] which, instead, relies on a very special construction of the (directed) sensing graph $\mathcal{G}$. The procedure of [18] cannot be, however, generalized to generic bearing rigid frameworks such as those considered in this venue. In order to cope with this problem, we now detail an extension of the localization algorithm introduced in [22] for obtaining a (decentralized) estimation of the relative orientations ${ }^{i} \boldsymbol{R}_{j}$ in presence of a generic bearing rigid graph and of nonstationary agents.

\section{B. Rigidity-based localization of time-varying bearing frameworks in $\mathbb{R}^{3} \times \mathcal{S}^{1}$}

Let $\hat{\boldsymbol{q}}=(\hat{\boldsymbol{p}}, \hat{\boldsymbol{\psi}})$ be an estimation of the true $\boldsymbol{q}$ and define the bearing estimation error as $\boldsymbol{e}_{L}(\boldsymbol{q}, \hat{\boldsymbol{q}})=\boldsymbol{\beta}_{\mathcal{G}}(\boldsymbol{q})-\boldsymbol{\beta}_{\mathcal{G}}(\hat{\boldsymbol{q}})$. Assuming $\boldsymbol{\beta}_{\mathcal{G}}(\boldsymbol{q}(t))=$ const, minimization of $\left\|\boldsymbol{e}_{L}\right\|$ can be obtained by this gradient descent based on the bearing rigidity matrix,

$$
\left[\begin{array}{c}
\dot{\hat{\boldsymbol{p}}} \\
\dot{\hat{\boldsymbol{\psi}}}
\end{array}\right]=k_{e} \boldsymbol{B}_{\mathcal{G}}^{\mathcal{W}}(\hat{\boldsymbol{q}})^{T} \boldsymbol{\beta}_{\mathcal{G}}(\boldsymbol{q}), \quad k_{e}>0
$$

Under the action of (11), the estimation $\hat{\boldsymbol{q}}(t)$ will converge towards a configuration equivalent to $\boldsymbol{q}$. Bearing rigidity of the framework $(\mathcal{G}, \boldsymbol{q})$ will also imply congruency with $\boldsymbol{q}$. Therefore, at convergence $\left(e_{L}=\mathbf{0}\right)$, the estimated $\hat{\boldsymbol{q}}$ will reach a configuration such that

$$
\left\{\begin{aligned}
\hat{\boldsymbol{p}} & =s\left(\boldsymbol{I}_{N} \otimes \boldsymbol{R}_{z}(\bar{\psi})\right) \boldsymbol{p}+\mathbf{1}_{N} \otimes \boldsymbol{t} \\
\hat{\boldsymbol{\psi}} & =\boldsymbol{\psi}+\mathbf{1}_{N} \bar{\psi}
\end{aligned}\right.
$$

for an arbitrary translation $t \in \mathbb{R}^{3}$, rotation angle $\bar{\psi} \in \mathcal{S}^{1}$ and scaling factor $s \in \mathbb{R}^{+}$. Any neighrboring pair can then replace the unknown ${ }^{i} \boldsymbol{R}_{j}$ with the estimated ${ }^{i} \hat{\boldsymbol{R}}_{j}=$ $\boldsymbol{R}_{z}\left(\hat{\psi}_{j}-\hat{\psi}_{i}\right)$ by exchanging the two estimates $\left(\hat{\psi}_{i}, \hat{\psi}_{j}\right)$ over local communication.

The estimator (11) is fully decentralized and only requires the bearings in $\boldsymbol{\beta}_{\mathcal{G}}(\boldsymbol{q})$ as measured quantities. However, the estimator (11) also assumes $\boldsymbol{\beta}_{\mathcal{G}}(\boldsymbol{q}(t))=$ const while the inter-agent relative bearings will be in general time-varying under the action of controller (10). ${ }^{2}$ Presence of a timevarying $\boldsymbol{\beta}_{\mathcal{G}}(\boldsymbol{q}(t))$ can clearly prevent convergence of the estimation error. This issue can be, however, addressed by adding to (11) the following feedforward term for taking into account the agent motion,

$$
\left[\begin{array}{c}
\dot{\hat{\boldsymbol{p}}} \\
\dot{\hat{\boldsymbol{\psi}}}
\end{array}\right]=k_{e} \boldsymbol{\mathcal { B }}_{\mathcal{G}}^{\mathcal{W}}(\hat{\boldsymbol{q}})^{T} \boldsymbol{\beta}_{\mathcal{G}}(\boldsymbol{q})+\left[\begin{array}{cc}
\operatorname{diag}\left(\boldsymbol{R}_{z}\left(\hat{\psi}_{i}\right)\right) & \mathbf{0} \\
\mathbf{0} & \boldsymbol{I}_{N}
\end{array}\right]\left[\begin{array}{c}
\boldsymbol{u} \\
\boldsymbol{w}
\end{array}\right]
$$

Proposition 2. If the initial estimation error $\left\|e_{L}\left(t_{0}\right)\right\|$ is small enough and $s=1$ then (13) will guarantee $\left\|\boldsymbol{e}_{L}(t)\right\| \rightarrow 0$ in case of time-varying bearings $\boldsymbol{\beta}_{\mathcal{G}}(\boldsymbol{q}(t)) \neq$ const.

\footnotetext{
${ }^{2}$ Indeed, $\boldsymbol{\beta}_{\mathcal{G}}(\boldsymbol{q}(t))=$ const only for stationary agents or for agents moving along the directions (6).
} 
Proof. The closed-loop dynamics of the estimation error is

$$
\begin{aligned}
\dot{\boldsymbol{e}}_{L} & =\boldsymbol{B}_{\mathcal{G}}(\boldsymbol{q})\left[\begin{array}{c}
\boldsymbol{u} \\
\boldsymbol{w}
\end{array}\right]-\boldsymbol{B}_{\mathcal{G}}^{\mathcal{W}}(\hat{\boldsymbol{q}})\left[\begin{array}{c}
\dot{\hat{\boldsymbol{p}}} \\
\dot{\hat{\boldsymbol{\psi}}}
\end{array}\right]= \\
& =-k_{e} \boldsymbol{B}_{\mathcal{G}}^{\mathcal{W}}(\hat{\boldsymbol{q}}) \boldsymbol{B}_{\mathcal{G}}^{\mathcal{W}}(\hat{\boldsymbol{q}})^{T} \boldsymbol{\beta}_{\mathcal{G}}(\boldsymbol{q})+\left(\boldsymbol{B}_{\mathcal{G}}(\boldsymbol{q})-\boldsymbol{B}_{\mathcal{G}}(\hat{\boldsymbol{q}})\right)\left[\begin{array}{c}
\boldsymbol{u} \\
\boldsymbol{w}
\end{array}\right] .
\end{aligned}
$$

The first term of (14) represents the (nominal) closed-loop dynamics of the constant bearing case, while the second term of (14) is a perturbation due to the agent motion. Since the nominal closed-loop dynamics is asymptotically stable [22], one can resort to the theory of perturbed systems [23] for analyzing the stability of (14). In particular, if the perturbation term can be shown to be vanishing with respect to the estimation error $e_{L}$, one can conclude local stability of the overall system (14) under mild conditions. Consider the $k$-th row of the body-frame rigidity matrix (8): this depends on the quantities $\boldsymbol{\beta}_{i j}, d_{i j}$ and ${ }^{i} \boldsymbol{R}_{j}$. By inspection one can then verify that, when $\boldsymbol{e}_{L}=\mathbf{0}$ (i.e., when (12) holds), ${ }^{i} \boldsymbol{R}_{j}={ }^{i} \hat{\boldsymbol{R}}_{j}, \boldsymbol{\beta}_{i j}=\hat{\boldsymbol{\beta}}_{i j}$ and $d_{i j}=s \hat{d}_{i j}$. Assuming $s=1$ in (12) then results in $\boldsymbol{e}_{L} \rightarrow \mathbf{0} \Longrightarrow \mathcal{B}_{\mathcal{G}}(\boldsymbol{q})-\mathcal{B}_{\mathcal{G}}(\hat{\boldsymbol{q}}) \rightarrow \mathbf{0}$ which concludes the proof.

A correct scale $(s=1)$ of the estimated formation $\hat{\boldsymbol{q}}$ is then necessary for properly compensating for the effects of the agent motion in the estimation dynamics. Since, as wellknown, the formation scale cannot be retrieved from only bearing measurements, we here exploit the presence of the single pair of agents, indexed as $\iota$ and $\kappa$, which is assumed able to also measure its relative distance $d_{\iota \kappa}$. One can then consider the following 'augmented' cost function,

$$
\frac{1}{2}\left(k_{e} \boldsymbol{e}_{L}^{T} \boldsymbol{e}_{L}+k_{d}\left(\hat{\boldsymbol{p}}_{\iota \kappa}^{T} \hat{\boldsymbol{p}}_{\iota \kappa}-d_{\iota \kappa}^{2}\right)^{2}\right), \quad k_{d}>0
$$

meant to enforce the constraint $\left\|\hat{\boldsymbol{p}}_{\iota \kappa}\right\|=\left\|\hat{\boldsymbol{p}}_{\iota}-\hat{\boldsymbol{p}}_{\kappa}\right\|=d_{\iota \kappa}$ in the estimated $\hat{\boldsymbol{q}}$. As shown in [22], minimization of (15) is obtained by complementing the update law (13) with the additional (decentralized) terms $\mp k_{d}\left(\hat{\boldsymbol{p}}_{\iota \kappa}^{T} \hat{\boldsymbol{p}}_{\iota \kappa}-d_{\iota \kappa}^{2}\right) \hat{\boldsymbol{p}}_{\iota \kappa}$ in the $\iota$-th and $\kappa$-th entries of $\dot{\hat{p}}$, respectively.

\section{Coordinated motions in the null-space of the bearing rigidity matrix}

As the final step, we address the fulfilment of the second control objective, that is, the implementation of the nullspace motions spanned by (6).

This can be achieved by realizing the world-frame velocity $\dot{\boldsymbol{q}}_{s}=\boldsymbol{n}_{1} \boldsymbol{\nu}+\boldsymbol{n}_{2} \lambda+\boldsymbol{n}_{3} w$ which imposes to the framework a common linear velocity $\boldsymbol{\nu} \in \mathbb{R}^{3}$, an expansion rate $\lambda \in \mathbb{R}$ about $\boldsymbol{O}_{\mathcal{W}}$, and a coordinated rotation with angular speed $w$ about a vertical axis passing through $\boldsymbol{O}_{\mathcal{W}}$. The corresponding body-frame velocities $\left(\boldsymbol{u}_{s}, \boldsymbol{w}_{s}\right)$ to be added to the formation control inputs $(\boldsymbol{u}, \boldsymbol{w})$ in (9) are then

$$
\left[\begin{array}{c}
\boldsymbol{u}_{s} \\
\boldsymbol{w}_{s}
\end{array}\right]=\left[\begin{array}{cc}
\operatorname{diag}\left(\boldsymbol{R}_{i}^{T}\right) & \mathbf{0} \\
\mathbf{0} & \boldsymbol{I}_{N}
\end{array}\right] \dot{\boldsymbol{q}}_{s} .
$$

While (16) realizes the second control objective, it is usually more interesting to implement an expansion rate and coordinated rotation about a specific point of interest attached to the formation itself, rather than about the (arbitrary) origin of the world frame $\boldsymbol{O}_{\mathcal{W}}$. For instance, an often convenient choice is to implement these motions relative to the formation centroid $\overline{\boldsymbol{p}}=\mathbf{1}_{N_{3}}^{T} \boldsymbol{p} / N$. This can be obtained by using as basis for $\mathcal{N}\left(\mathcal{B}_{\mathcal{G}}^{\mathcal{W}}(\boldsymbol{q})\right)$ the set $\left\{\boldsymbol{n}_{1}, \boldsymbol{n}_{2}-\boldsymbol{n}_{1} \overline{\boldsymbol{p}}, \boldsymbol{n}_{3}-\boldsymbol{n}_{1} \boldsymbol{S} \overline{\boldsymbol{p}}\right\}$ which eventually results in the $i$-th agent velocity commands

$$
\left\{\begin{array}{l}
\boldsymbol{u}_{s_{i}}=\boldsymbol{R}_{i}^{T}\left(\boldsymbol{\nu}+\lambda\left(\boldsymbol{p}_{i}-\overline{\boldsymbol{p}}\right)+w \boldsymbol{S}\left(\boldsymbol{p}_{i}-\overline{\boldsymbol{p}}\right)\right) \\
w_{s_{i}}=w
\end{array}\right.
$$

An actual implementation of (17) would require the (non-available) quantities $\left(\boldsymbol{p}_{i}, \psi_{i}, \overline{\boldsymbol{p}}\right)$ : exploiting the estimator (13), each agent can replace the true $\left(\boldsymbol{p}_{i}, \psi_{i}\right)$ with the estimated $\left(\hat{\boldsymbol{p}}_{i}, \hat{\psi}_{i}\right)$. The corresponding average $\hat{\overline{\boldsymbol{p}}}=\mathbf{1}_{N_{3}}^{T} \hat{\boldsymbol{p}} / N$ can then be obtained by resorting to any distributed averaging filtering technique such as the well-known PI average consensus filter (PI-ACE) ${ }^{3}$ [26].

It is worth noting that knowledge of the correct scale factor $(s=1)$ in the estimated $\hat{\boldsymbol{q}}$ is not required for implementing the null-space motions associated to vectors $\boldsymbol{n}_{1}$ and $\boldsymbol{n}_{2}$ (translation and expansion), but it is instead required for correctly implementing the coordinated rotation associated to vector $\boldsymbol{n}_{3}$. Indeed, $\boldsymbol{n}_{1}$ does not depend on $\boldsymbol{p}$ and $\boldsymbol{n}_{2}$ is homogeneous in $\boldsymbol{p}$, with its direction thus unaffected by any scaling of the agent positions. This is, however, not the case for vector $\boldsymbol{n}_{3}$ which is not homogeneous in $\boldsymbol{p}$. Similarly to the estimation case, if a distance measurement is not available for fixing the scale of $\hat{\boldsymbol{q}}$, the coordinated rotation will not be exactly implemented.

\section{Discussion}

We conclude by emphasizing that, as stated at the beginning of the section, the proposed control/estimation scheme does not require a special topology for the interaction graph (besides being bearing-rigid); the bearing controller (10), the localization algorithm (13), and the null-space motions (17) have the same (decentralized) expression for all agents only as a function of the measured bearings and body-frame linear/angular velocities. The only exception is the inclusion of the distance measurement $d_{\iota \kappa}$ which adds an additional control term to agents $\iota$ and $\kappa$. In this sense, we believe that the present work represents a significant generalization of the strategy reported in [18] which, to the best of our knowledge, is the closest related work to our setting, and relied on a much more constrained design of the agent group.

We also note that the correct formation scale could be retrieved without assuming the presence of an (additional) distance measurement $d_{\iota \kappa}$ (and, thus, presence of two 'special agents' in the group). Indeed, the unknown robot inter-distances could be estimated online by processing the measured inter-robot bearings and the (known) robot own motions similarly to what done in the context of scale estimation for point features [27], [28]. In this respect, the

\footnotetext{
${ }^{3}$ Indeed, given a (time-varying) vector quantity $\boldsymbol{x}(t) \in \mathbb{R}^{N}$ with each component $x_{i}(t)$ locally available to agent $i$, the PI-ACE filter allows every agent to distributedly build an estimation converging to the average $\bar{x}(t)=$ $\sum_{i=1}^{N} x_{i}(t) / N$ with a tunable dynamics that can be made faster than the underlying dynamics of each agent in the system, see also [7], [24], [25] for some applications in the context of multi-robot distributed control.
} 


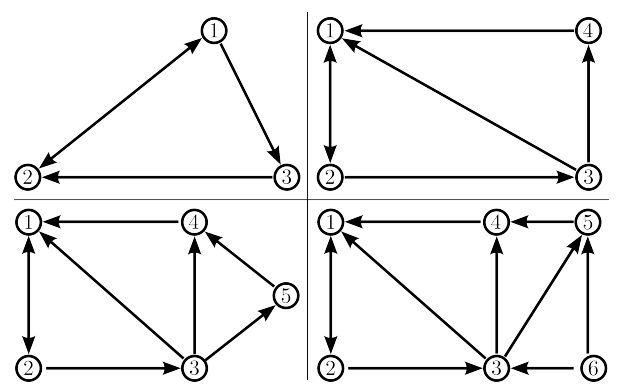

Fig. 1: Possible minimal bearing rigid topologies for $N \in$ $\{3,4,5,6\}$ (note that some arrows are bi-directional)

work [29] proposes an active scale estimation strategy for bearing formations of quadrotor UAVs.

We finally wish to briefly discuss the practical implications of requiring directed (bearing) rigidity for the robot formation (which, as often stated, is an underlying necessary condition of the proposed machinery). As well-known, minimal rigidity requires presence of a $|\mathcal{E}|=O(N)$ number of edges (i.e., of inter-robot measurements/constraints) in the framework vs. the quadratic complexity of the complete (directed) graph $\mathcal{K}_{N}$ (for which the complexity would be $\left.N(N-1)=O\left(N^{2}\right)\right)$. As illustration, Fig. 1 shows some possible minimal bearing rigid topologies for the case of $N \in$ $\{3 \ldots 6\}$ agents for which $|\mathcal{E}|=4,6,8,10$, respectively.

\section{SIMULATION RESULTS}

We now present some simulation results involving $N=6$ quadrotors. The simulation is run by considering the full dynamics of quadorotor UAVs simulated via the 3D physical simulator V-REP. In this case, the robustness of the bearing controller is tested against the discrepancies between the nominal agent (1) and the actual quadrotor flight dynamics, as well as against noise and discretization in the measured bearings (which are sampled at $60 \mathrm{~Hz}$ for mimicking an actual onboard camera).

The initial configuration $\boldsymbol{q}\left(t_{0}\right)$ and estimated $\hat{\boldsymbol{q}}\left(t_{0}\right)$ were generated by adding to the desired $\boldsymbol{q}_{d}$ a uniformly distributed random perturbation of amplitude $1 \mathrm{~m}$ for the positions and $120 \mathrm{deg}$ for the orientation. A graph $\mathcal{G}$ with $|\mathcal{E}|=20$ directed edges was then randomly generated under the constraint of guaranteeing bearing rigidity at $\boldsymbol{q}\left(t_{0}\right), \hat{\boldsymbol{q}}\left(t_{0}\right)$ and $\boldsymbol{q}_{d}$, and the following gains were used: $k_{c}=1$ in (10), $k_{e}=5$ in (13) and $k_{d}=10$ in (15). Finally, in all simulations the null-space velocity commands (17) were activated, for convenience of illustration, only after convergence to the desired formation $\boldsymbol{b}_{\mathcal{G}}^{d}$ : the five coordinated motions were first actuated one at the time and then all together.

Figures 2-3 report the simulation results. In particular, the bottom Fig. 2(a) shows the behavior of $\left\|e_{F}(t)\right\|$ (the bearing control error, solid blue line) and of $\left\|e_{L}(t)\right\|$ (the estimation error, solid red line), while the top Fig. 2(a) depicts the five null-space velocity commands $(\boldsymbol{\nu}, \lambda, w)$ in (17). It is then possible to verify how $(i)$ both the control and estimation bearing errors converge to zero (despite their initial large value and despite the presence of time-varying bearings $\boldsymbol{\beta}(\boldsymbol{q}(t)))$ and how $(i i)$ the implementation of the coordinated

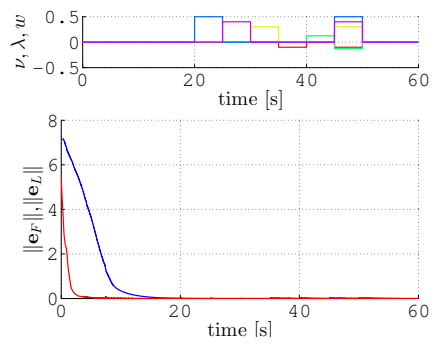

(a)

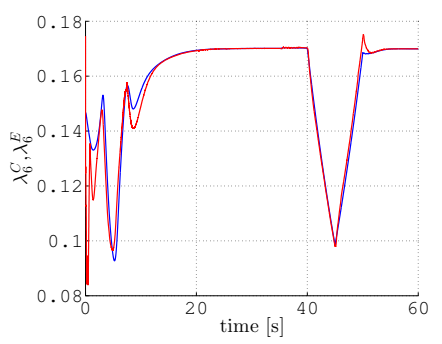

(b)
Fig. 2: Results of the simulation. (a)-top: behavior of the five nullspace motion commands $\boldsymbol{\nu}(t)$ (blue, purple, yellow) $\lambda(t)$ (green) and $w(t)$ (red). (a)-bottom: behavior of the bearing control error $\left\|\boldsymbol{e}_{F}(t)\right\|$ and of the localization control error $\left\|\boldsymbol{e}_{L}(t)\right\|$. (b): behavior of the rigidity eigenvalues $\lambda_{6}^{C}(t)$ (control - blue) and $\lambda_{6}^{E}(t)$ (estimation - red)

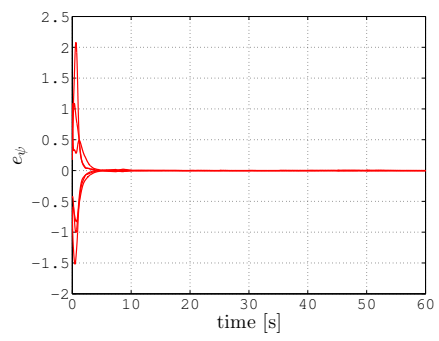

(a)

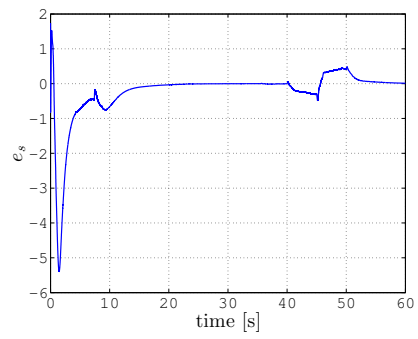

(b)
Fig. 3: Results of the first simulation. (a): behavior of the orientation estimation error $\boldsymbol{e}_{\psi}(t)$. (b): behavior of the formation scale error $e_{s}(t)$

motions (17) has no disturbing effect on the bearing errors (as expected). Let $\lambda_{6}(\boldsymbol{q}) \geq 0$ represent the sixth smallest eigenvalue of the square matrix $\left(\boldsymbol{B}_{\mathcal{G}}^{\mathcal{W}}(\boldsymbol{q})\right)^{T} \boldsymbol{B}_{\mathcal{G}}^{\mathcal{W}}(\boldsymbol{q})$. Since for an infinitesimal rigid framework it holds $\operatorname{rank}\left(\boldsymbol{B}_{\mathcal{G}}^{\mathcal{W}}(\boldsymbol{q})\right)=$ $4 N-5$, the quantity $\lambda_{6}(\boldsymbol{q})$ can be taken as a measure of the framework bearing rigidity $\left(\lambda_{6}(\boldsymbol{q})>0\right.$ iff $(\mathcal{G}, \boldsymbol{q})$ is infinitesimal rigid and $\lambda_{6}(\boldsymbol{q})=0$ otherwise). Figure 2(b) then reports the behavior of $\lambda_{6}^{C}(t)=\lambda_{6}(\boldsymbol{q}(t))$ and $\lambda_{6}^{E}(t)=$ $\lambda_{6}(\hat{\boldsymbol{q}}(t))$, that is, the rigidity measures for the 'control' framework $(\mathcal{G}, \boldsymbol{q})$ and the 'estimation' framework $(\mathcal{G}, \hat{\boldsymbol{q}})$. One can then check how both frameworks remained rigid during motion, thus confirming congruency between $\boldsymbol{q}$ and $\boldsymbol{q}_{d}$ (correct agent formation), and between $\boldsymbol{q}$ and $\hat{\boldsymbol{q}}$ (correct agent localization).

As an additional measure of the localization performance, we considered the quantity $\boldsymbol{e}_{\psi}=\left(\boldsymbol{I}_{N}-\mathbf{1}_{N} \mathbf{1}_{N}^{T} / N\right)(\boldsymbol{\psi}-\hat{\boldsymbol{\psi}})$ : this represents the disagreement between the orientation estimation error and its mean value, and it should vanish in presence of a correct localization ${ }^{4}$ as, indeed, reported in Fig. 3(a). A converging $\boldsymbol{e}_{\psi}(t)$ then allows to correctly compute the missing terms ${ }^{i} \boldsymbol{R}_{j}$ in the bearing controller (10). Finally, Fig. 3(b) depicts the behavior of the 'formation scale error' defined as $e_{s}(t)=\left\|\boldsymbol{p}(t)-\mathbf{1}_{N} \otimes \overline{\boldsymbol{p}}(t)\right\|-\| \hat{\boldsymbol{p}}(t)-\mathbf{1}_{N} \otimes$ $\hat{\overline{\boldsymbol{p}}}(t) \|$ which, again, converges to zero, as expected, thanks to the additional distance constraint in (15).

It is worth noting that the 'distortions' present in Fig. 3(b)

${ }^{4}$ Indeed, a correct localization implies that $\hat{\boldsymbol{\psi}}(t) \rightarrow \boldsymbol{\psi}(t)+\mathbf{1}_{N} \bar{\psi}$ (see (12)). 


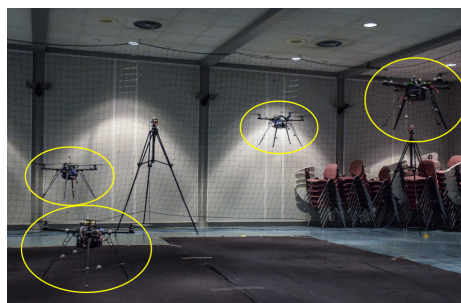

(a)

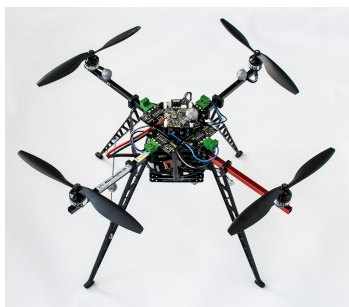

(b)
Fig. 4: The flying arena (a) and our quadrotor platform (b)

are mainly due to the higher-order quadrotor dynamics neglected by model (1) which, roughly speaking, introduces an unmodeled lag between commanded and actual velocities. The proposed control strategy is nevertheless robust enough for coping with these model inaccuracies.

\section{EXPERIMENTAL RESULTS}

This section discusses the experiment conducted for validating the formation controller described in Sect. III (the reader is also referred to the attached video).

For the experiment illustrated in this section we used a group of four quadrotor UAVs MK-Quadro from MikroKopter (see Fig. 4(b)). The usual MK-Quadro setup was extended with an ODROID-XU4 Linux Computer running ROS and the TeleKyb framework [30] for interfacing our bearing control algorithm with the MK-Quadro low-level controller. A Vicon motion capture system was employed for reconstructing the body-frame bearing measurements $\boldsymbol{\beta}_{i j}$ that would have been obtained by an onboard camera running at $60 \mathrm{~Hz}$. The experiments were performed in our flying arena which has a volume of $6.5 \mathrm{~m} \times 5 \mathrm{~m} \times 3 \mathrm{~m}$ (see Fig. 4(a)).

The reported experiment followed a pattern similar to the previous simulation results: $(i)$ regulation towards a desired bearing formation, ( $i i)$ actuation of the null-space motions (17), (iii) regulation towards a different desired bearing formation, ( $i v)$ actuation of the null-space motions (17). Additionally, we implemented, at every 6 seconds, a random switch among all the possible rigid topologies for the sensing graph in order to show the robustness of our approach also against possible topology changes during motion.

Figures 5-6 report the results of the experiment. The UAV formation starts far from the desired configuration but, after about 20 seconds, the norm of the formation control error $\left\|e_{F}(t)\right\|$ drops below $4 \%$ of its initial value (Fig. 5(a)). On the other hand, convergence of the estimator error $\left\|e_{L}(t)\right\|$ is quite fast even though the initial estimated $\hat{\boldsymbol{q}}\left(t_{0}\right)$ was generated by adding to the real $\boldsymbol{q}\left(t_{0}\right)$ a uniformly distributed random perturbation of amplitude $1.5 \mathrm{~m}$ for the positions $\boldsymbol{p}\left(t_{0}\right)$ and $80 \mathrm{deg}$ for the orientations $\boldsymbol{\psi}\left(t_{0}\right)$. Convergence of the estimated $\hat{\boldsymbol{q}}(t)$ towards a configuration congruent with $\boldsymbol{q}(t)$ (and with the correct scale) can also be appreciated in Fig. 6 where the orientation estimation error $\boldsymbol{e}_{\psi}(t)$ and the formation scale error $e_{s}(t)$ are shown. One can then verify, again, how a consistent estimation of the orientations $\hat{\psi}$ and

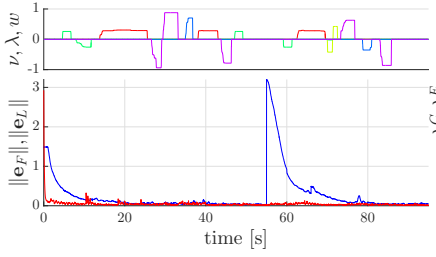

(a)

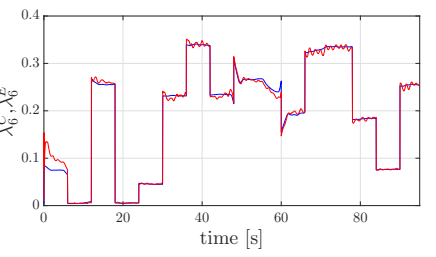

(b)
Fig. 5: Results of the experiment. (a)-top: behavior of the five null-space motion commands $\boldsymbol{\nu}(t)$ (blue, purple, yellow) $\lambda(t)$ (green) and $w(t)$ (red). (a)-bottom: behavior of the bearing control error $\left\|\boldsymbol{e}_{F}(t)\right\|$ and of the localization control error $\left\|\boldsymbol{e}_{L}(t)\right\|$. (b): behavior of the rigidity eigenvalues $\lambda_{6}^{C}(t)$ (control - blue) and $\lambda_{6}^{E}(t)$ (estimation - red)

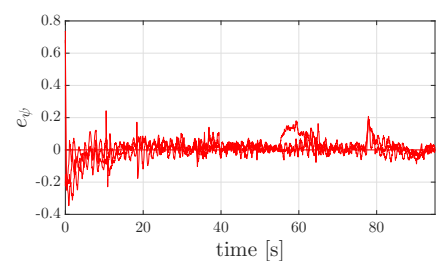

(a)

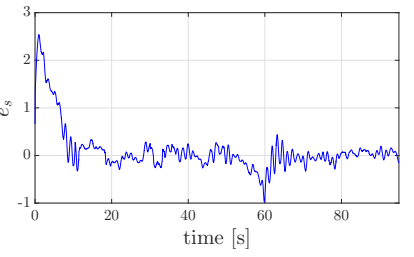

(b)
Fig. 6: Results of the experiment. (a): behavior of the orientation estimation error $\boldsymbol{e}_{\psi}(t)$. (b): behavior of the formation scale error $e_{s}(t)$

of the formation scale $s$ could be obtained despite the (unavoidable) non-idealities present in any real implementation.

Finally, as stated before, the underlying graph $\mathcal{G}$ switches randomly at every 6 seconds across all the possible rigid topologies for the $N=4$ quadrotors (in particular, we allowed switches among graphs with $|\mathcal{E}| \in\{6,7, \ldots, 12\}$ ). Figure 5(b) shows the behavior of the rigidity measures $\lambda_{6}^{C}(t)$ and $\lambda_{6}^{E}(t)$ which 'jump' at every 6 seconds (as expected) because of the topology switches. Nevertheless, these topology changes (and associated increases/decreases of the control/estimation framework rigidity) did not negatively affected the overall performance of the proposed bearing control strategy.

\section{CONCLUSIONS}

In this paper we have considered the problem of devising a decentralized control strategy for controlling a group of quadrotor UAVs able to measure relative bearings in their own body frames. In particular, we considered two control objectives: $(i)$ stabilization of the quadrotor formation towards a desired bearing configuration, and $(i i)$ steering of the whole formation along the motion directions in the null-space of the bearing rigidity matrix. To this end, a suitable $\mathbb{R}^{3} \times \mathcal{S}^{1}$ extension and combination of the $S E(2)$ directed bearing rigidity control/localization algorithms introduced in [19], [22] has been developed for the case of non-stationary agents, together with a full explicit characterization of the nullspace of the bearing rigidity matrix. This allowed to devise a decentralized bearing controller able to meet the two control objectives without the need of a common reference frame for the agent group, nor the requirement of reciprocal bearing measurements (i.e., of an undirected measurement topology). Simulation and experimental results on real quadrotors have 
been proposed to illustrate the various features of the approach.

We are currently working towards a fully-onboard implementation of the proposed ideas by equipping the UAVs with onboard cameras for retrieving relative bearings and estimating the quadrotor body-frame linear/angular velocities $\left(\boldsymbol{u}_{i}, w_{i}\right)$ (thus freeing from the need of an external motion capture system). On the theoretical side, we are interested in extending the ideas of [7] for dealing with the issue of bearing rigidity maintenance in presence of possible occlusions or loss of tracking of neighboring UAVs because of limited camera fov. This would then allow the quadrotor group to flexibily navigate in cluttered environment under the (controlled) possibility of losing/gaining neighbors while ensuring a minimum level of bearing rigidity for the formation. Another interesting extension is the (decentralized) integration of Structure from Motion (SfM) schemes, such as [29], able to recover online the missing scale information by processing the measured bearings and known agent motion (and, thus, avoiding the requirement of a special agent pair able to additionally measure its inter-distance).

\section{APPENDIX}

The proof of Prop. 1 that vectors $\left[\mathbf{1}_{N_{3}}^{T} \mathbf{0}^{T}\right]^{T}$ and $\left[\boldsymbol{p}^{T} \mathbf{0}^{T}\right]^{T}$ belong to $\mathcal{N}\left(\mathcal{B}_{\mathcal{G}}^{\mathcal{W}}\right)$ can be found in [19], [22]. The explicit expression for the last null-space vector in (6) (not present in [19], [22]) can be shown as follows: consider the $k$-th element of $\mathcal{B}_{\mathcal{G}}^{\mathcal{W}}\left[\begin{array}{l}p^{\perp} \\ \mathbf{1}_{N}\end{array}\right]$, which has the following expression,

$$
\begin{aligned}
& \frac{\boldsymbol{P}_{i j} \boldsymbol{R}_{i}^{T} \boldsymbol{S}\left(\boldsymbol{p}_{j}-\boldsymbol{p}_{i}\right)}{d_{i j}}-\boldsymbol{S} \boldsymbol{\beta}_{i j}=\boldsymbol{P}_{i j} \boldsymbol{R}_{i}^{T} \boldsymbol{S} \boldsymbol{R}_{i} \frac{\boldsymbol{R}_{i}^{T}\left(\boldsymbol{p}_{j}-\boldsymbol{p}_{i}\right)}{d_{i j}} \\
& -\boldsymbol{S} \boldsymbol{\beta}_{i j}=\boldsymbol{P}_{i j} \boldsymbol{S} \boldsymbol{\beta}_{i j}-\boldsymbol{S} \boldsymbol{\beta}_{i j}=\boldsymbol{S} \boldsymbol{\beta}_{i j}-\boldsymbol{S} \boldsymbol{\beta}_{i j}=\mathbf{0}
\end{aligned}
$$

where the properties $\boldsymbol{R}_{i}^{T} \boldsymbol{S} \boldsymbol{R}_{i}=\boldsymbol{S}$ and $\boldsymbol{P}_{i j} \boldsymbol{S} \boldsymbol{\beta}_{i j}=\boldsymbol{S} \boldsymbol{\beta}_{i j}$ have been used (the last one exploits the fact that $\boldsymbol{S} \boldsymbol{\beta}_{i j} \perp$ $\left.\boldsymbol{\beta}_{i j}\right)$.

\section{ACKNOWLEDGMENTS}

This research was supported by the ANR, Project ANR14-CE27-0007 SenseFly.

\section{REFERENCES}

[1] R. Olfati-Saber, J. A. Fax, and R. M. Murray, "Consensus and cooperation in networked multi-agent systems," Proc. of the IEEE, vol. 1, no. 95, pp. 215-233, 2007.

[2] J. Cortés, "Global and robust formation-shape stabilization of relative sensing networks," Automatica, vol. 12, no. 45, pp. 2754-2762, 2009.

[3] E. Montijano, D. Zhou, M. Schwager, and C. Sagues, "Distributed formation control without a global reference frame," in 2014 ACC, Portland, OR, Jun. 2014, pp. 3862-3867.

[4] B. D. O. Anderson, Y. Changbin, B. Fidan, and J. Hendrickx, "Rigid graph control architectures for autonomous formations," IEEE Control Systems Magazine, vol. 6, no. 28, pp. 48-63, 2008.

[5] L. Krick, M. E. Broucke, and B. A. Francis, "Stabilisation of infinitesimally rigid formations of multi-robot networks," Int. J. of Control, vol. 82, no. 3, pp. 423-439, Mar. 2009.

[6] H. Garcia de Marina, M. Cao, and B. Jayawardhana, "Controlling rigid formations of mobile agents under inconsistent measurements," IEEE Trans. on Robotics, vol. 31, no. 1, pp. 31-39, Feb 2015.

[7] D. Zelazo, A. Franchi, H. H. Bülthoff, and P. Robuffo Giordano, "Decentralized Rigidity Maintenance Control with Range-only Measurements for Multi-Robot Systems," Int. J. of Robotics Research, vol. 34, no. 1, pp. 105-128, 2015.
[8] A. N. Bishop, I. Shames, and B. D. Anderson, "Stabilization of rigid formations with direction-only constraints," in 2011 IEEE CDC and ECC, Dec. 2011, pp. 746-752.

[9] T. Eren, "Formation shape control based on bearing rigidity," Int. J. of Control, vol. 9, no. 85, pp. 1361-1379, 2012.

[10] A. Franchi and P. Robuffo Giordano, "Decentralized control of parallel rigid formations with direction constraints and bearing measurements,' in 2012 IEEE CDC, Maui, HI, Dec. 2012, pp. 5310-5317.

[11] A. Cornejo, A. J. Lynch, E. Fudge, S. Bilstein, M. Khabbazian, and J. McLurkin, "Scale-free coordinates for multi-robot systems with bearing-only sensors," The Int. J. of Robotics Research, vol. 12, no. 32, pp. 1459-1474, 2013.

[12] S. Zhao and D. Zelazo, "Bearing rigidity and almost global bearingonly formation stabilization," IEEE Transactions on Automatic Control, vol. 61, no. 5, pp. 1255-1268, 2016.

[13] — " "Translational and scaling formation maneuver control via a bearing-based approach," IEEE Transactions on the Control of Network Systems (early access), vol. PP, pp. 1-10, 2015.

[14] T. Eren, "Using angle of arrival (bearing) information for localization in robot networks," Turkish J. of Elec. Eng. \& Comput. Sci., vol. 15, no. 2, pp. 169-186, 2007.

[15] G. C. Calafiore, L. Carlone, and M. Wei, "A distributed gradient method for localization of formations using relative range measurements," in 2010 IEEE Int. Symp. on Computer-Aided Control System Design, Yokohama, Japan, Sep. 2010, pp. 1146-1151.

[16] I. Shames, A. N. Bishop, and B. D. O. Anderson, "Analysis of noisy bearing-only network localization," IEEE Trans. on Automatic Control, vol. 1, no. 58, pp. 247-252, 2013.

[17] R. Tron, L. Carlone, F. Dellaert, and K. Daniilidis, "Rigid components identification and rigidity enforcement in bearing-only localization using the graph cycle basis," in 2015 ACC, Chicago, IL, Jul. 2015 , pp. 3911-3918.

[18] A. Franchi, C. Masone, V. Grabe, M. Ryll, H. H. Bülthoff, and P. Robuffo Giordano, "Modeling and control of UAV bearingformations with bilateral high-level steering," The Int. J. of Robotics Research, Special Issue on 3D Exploration, Mapping, and Surveillance, vol. 31, no. 12, pp. 1504-1525, 2012.

[19] D. Zelazo, P. Robuffo Giordano, and A. Franchi, "Bearing-Only Formation Control Using an SE(2) Rigidity Theory," in 2015 IEEE CDC, Osaka, Japan, Dec. 2015, pp. 6121-6126.

[20] C. D. Godsil and G. Royle, Algebraic Graph Theory. Springer, 2001.

[21] T. Eren, W. Whiteley, A. S. Morse, P. N. Belhumeur, and B. D. Anderson, "Sensor and Network Topologies of Formations with Direction, Bearing, and Angle Information between Agents," in 2003 IEEE CDC Maui, HI, Dec. 2003, pp. 3064-3069.

[22] D. Zelazo, A. Franchi, and P. Robuffo Giordano, "Rigidity Theory in $S E(2)$ for Unscaled Position Estimation using only Bearing Measurements," in 2014 ECC, Strasbourg, France, Jun. 2014, pp. 2703-2708.

[23] H. K. Khalil, Nonlinear Systems. Prentice Hall PTR, 2002.

[24] P. Yang, R. A. Freeman, G. J. Gordon, K. M. Lynch, S. S. Srinivasa, and R. Sukthankar, "Decentralized estimation and control of graph connectivity for mobile sensor networks," Automatica, vol. 46, no. 2 , pp. 390-396, 2010

[25] P. Robuffo Giordano, A. Franchi, C. Secchi, and H. H. Bülthoff, "A passivity-based decentralized strategy for generalized connectivity maintenance," The Int. J. of Robotics Research, vol. 32, no. 3, pp. 299-323, 2013.

[26] R. A. Freeman, P. Yang, and K. M. Lynch, "Stability and convergence properties of dynamic average consensus estimators," in 2006 IEEE $C D C$, San Diego, CA, Dec. 2006, pp. 338-343.

[27] A. De Luca, G. Oriolo, and P. Robuffo Giordano, "Feature depth observation for image-based visual servoing: Theory and experiments,' Int. J. of Robotics Research, vol. 27, no. 10, pp. 1093-1116, 2008.

[28] R. Spica, P. Robuffo Giordano, and F. Chaumette, "Active Structure from Motion: Application to Point, Sphere and Cylinder," IEEE Trans. on Robotics, vol. 30, no. 6, pp. 1499-1513, 2014.

[29] R. Spica and P. Robuffo Giordano, "Active Decentralized Scale Estimation for Bearing Based Localization," in 2016 IEEE IROS, Daejeon, Korea, Oct. 2016.

[30] V. Grabe, M. Riedel, H. H. Bulthoff, P. R. Giordano, and A. Franchi, "The telekyb framework for a modular and extendible ros-based quadrotor control," in 2013 IEEE ECMR, Barcelona, Spain, Sep. 2013 , pp. 19-25. 